\title{
Reduced ubiquitin-specific protease 9X expression induced by RNA interference inhibits the bioactivity of hepatocellular carcinoma cells
}

\author{
HUIWEN HU ${ }^{1}$, CHENGYONG TANG ${ }^{2}$, QINGHU JIANG ${ }^{1}$, WEI LUO ${ }^{1}$, \\ JIMING LIU ${ }^{1}$, XUFU WEI ${ }^{1}$, RUI LIU ${ }^{1}$ and ZHONGJUN WU ${ }^{1}$ \\ Departments of ${ }^{1}$ Hepatobiliary Surgery and ${ }^{2}$ Clinical Pharmacology, The First Affiliated Hospital of \\ Chongqing Medical University, Chongqing 400016, P.R. China
}

Received June 18, 2014; Accepted February 24, 2015

DOI: $10.3892 / \mathrm{ol} .2015 .3152$

\begin{abstract}
Ubiquitin-specific protease 9X (USP9X) is crucial in many tumor types, but not in hepatocellular carcinoma (HCC). The current study aimed to examine the effects of RNA interference on USP9X expression, and subsequently on the bioactivity of HCC SMMC7721 and HepG2 cells. The protein expression of USP9X in SMMC7721, HepG2 and normal human liver cell line L02 at the cellular level was determined by western blot analysis; USP9X was knocked down by small interfering RNA (siRNA) in HCC SMMC7721 and HepG2 cells. In vitro cell viability was assessed by MTT assay, apoptosis was determined by flow cytometry (FCM) and cell migration was evaluated by Transwell assays. The protein expression of USP9X in SMMC7721 and HepG2 were both significantly higher than that in L02 $(\mathrm{P}<0.01)$. The results of western blot demonstrated that the USP9X-siRNA could efficiently inhibit USP9X expression when compared with that of the negative control $(\mathrm{NC})$ group $(\mathrm{P}<0.01)$ and MTT assay demonstrated that cell proliferation in USP9X-blocked cells was significantly reduced when compared with that of the NC group $(\mathrm{P}<0.01)$. The results of FCM revealed that apoptosis was significantly increased in USP9X-blocked cells when compared with that of the $\mathrm{NC}$ group $(\mathrm{P}<0.01)$. The results of transwell assay showed that cell migration was significantly inhibited in USP9X-blocked cells when compared with that of the NC group $(\mathrm{P}<0.01)$. These results show that expression of USP9X is upregulated in hepatoma cells SMMC7721 and HepG2, and that downregulating USP9X by siRNA may induce cell apoptosis, inhibit cell growth and cell migration in the HCC SMMC7721 and HepG2 cell lines. USP9X may
\end{abstract}

Correspondence to: Professor Zhongjun Wu, Department of Hepatobiliary Surgery, The First Affiliated Hospital of Chongqing Medical University, 1 Youyi Road, Chongqing 400016, P.R. China E-mail: wzjtcy@126.com

Key words: apoptosis, growth, hepatocellular carcinoma, migration, RNA interference, ubiquitin-specific protease 9X therefore be a potential target for HCC treatment and early detection.

\section{Introduction}

Hepatocellular carcinoma (HCC), as one of the most malignant cancers worldwide, causes $>500,000$ mortalities worldwide every year and has an extremely poor prognosis $(1,2)$. Currently, chemotherapy, liver transplantation, surgical resection, and local ablation are used for the treatment of HCC, which is dependent on the stage of HCC (3). HCC leads to a high mortality due to the lack of reliable early detection (4). Therefore, a reliable target is urgently required for the development of early detection and effective treatment techniques for HCC.

Ubiquitin-specific protease 9X (USP9X) is a X-linked ubiquitin specific peptidase which belongs to the ubiquitin-specific protease family (5). USP9X serves as a deubiquitinase and effectively regulates the proliferation, adhesion and signal transduction of cells, therefore is crucial in controlling proteasome activity in organogenesis, transcriptional regulation and tumorigenesis (6-9). Previous studies have demonstrated an association between USP9X and lung, colon (5) and breast cancer (10), and lymphoma (11). Specifically, previous studies have shown that high USP9X expression results in a poor prognosis in lymphoma and lung cancer (10-14). Thus, we hypothesize that high USP9X expression is associated with the growth and metastasis of tumor cells.

However, to date, the role of USP9X in HCC has not been reported. In the present study, the expression of USP9X was blocked in SMMC7721 and HepG2 cells using specific small interfering RNA (siRNA) to explore the potential influence of USP9X in HCC cell lines.

\section{Materials and methods}

Cell culture. The human HCC cell lines SMMC7721 and HepG2, and human liver cell line, L02 were purchased from American Type Culture Collection (Manassas, VA, USA). Cells were cultured in RPMI-1640 (Invitrogen Life 
Table I. Sequences of USP9X-siRNA and negative control.

\begin{tabular}{ll}
\hline Name & \multicolumn{1}{c}{ Sequences } \\
\hline USP9X-siRNA & Sense (5'-3') AGAAAUCGCUGGUAUAAAUUU \\
Negative control & Antisense (5'-3') AAAUUUAUACCAGCGAUUUCU \\
& Sense (5'-3') UUCUCCGAACGUGUCACGUTT \\
& Antisense (5'-3') ACGUGACACGUUCGGAGAGTT \\
\hline
\end{tabular}

USP9X, ubiquitin specific protease 9X.

Technologies, Carlsbad, CA, USA) supplemented with $10 \%$ fetal bovine serum (FBS; Invitrogen Life Technologies) at $37^{\circ} \mathrm{C}$ in a humidified atmosphere of $5 \% \mathrm{CO}_{2}$.

RNA interference. USP9X-specific siRNA (USP9X-siRNA) and a negative control (NC group) with no significant homology with human gene sequences were purchased from Gene Pharma (Shanghai, China). The sequences of USP9X-siRNA and NC are shown in Table I. Cells were seeded into a 6-well plate for $24 \mathrm{~h}$ at a density of $5 \times 10^{4}$ cells/well. In each well, $20 \mu \mathrm{M}$ USP9X-siRNA or negative control were transfected using Lipofectamine 2000 (Invitrogen Life Technologies) according to the manufacture's instruction.

Antibodies. The anti-USP9X (1:1,500; cat. no. 66026-1-Ig) antibody was purchased from Proteintech (Chicago, IL, USA). The anti $\beta$-tubulin antibodies (1:5,000; cat. no. KM9003) were purchased from Tianjin Sungene Biotechnology Company (Tianjin, China).

Western blot analysis. Total protein was extracted using the Total Protein Extraction Kit (Beyotime Institute of Biotechnology, Shanghai, China); USP9X protein was separated by $6 \%$ SDS-PAGE and then transferred to nitrocellulose membranes (Pierce, Rockford, USA) and incubated at $37^{\circ} \mathrm{C}$ for $1 \mathrm{~h}$ with the indicated primary and horseradish peroxidase-secondary antibodies (cat. no. LK2001; Beyotime Institute of Biotechnology). The labeled proteins were detected using chemiluminescent agents. Images were collected by Image-Pro plus software 6.0 (Media Cybernetics, Silver Spring, MD, USA). The relative levels of the target protein are represented as the density ratio versus $\beta$-tubulin. Independent experiments were repeated three times for each sample and the relative expression levels of protein were normalized to the endogenous reference gene $\beta$-tubulin and analyzed by using $2^{-\triangle \Delta C \mathrm{~T}}$ method (15).

Flow cytometry assay (FCM) for apoptosis. Annexin-V/propidium iodide (PI) double assay was performed using the Annexin V-FITC Apoptosis Detection kit (Nanjing KeyGen Biotechnology, Nanjing, China). Cells were released from the culture dish with trypsin and washed twice with phosphate buffered saline (PBS; BoPei Biotech Co. Ltd., Chongqing, China). Cells $\left(1 \times 10^{6}\right)$ were resuspended in $500 \mu$ l binding buffer and stained with $5 \mu$ l FITC-labeled Annexin-V according to the manufacturer's instructions. Following this, $5 \mu \mathrm{l}$ PI was added and allowed to incubate with the cells for $10 \mathrm{~min}$ at room temperature in the dark. After one wash with PBS, cells were subjected to FCM analysis using BD FACSCalibur (BD Biosciences, San Jose, CA, USA). The data were analyzed using CellQuest data acquisition and analysis software (version 5.1; BD Biosciences).

MTT assay. Cells were plated in 96-well plates at a density of $\sim 4 \times 10^{3}$ cells/well. Following treatment, the plates were incubated in a $37^{\circ} \mathrm{C}$ humidified incubator for the time periods indicated below. To assess cell viability, the MTT assay was performed according to the manufacturer's instructions (Sigma-Aldrich, St Louis, MO, USA). In brief, $20 \mu 1$ of MTT reagent $(5 \mathrm{mg} / \mathrm{ml})$ was added to each well, and the cells were incubated for a further $4 \mathrm{~h}$ at $37^{\circ} \mathrm{C}$, followed by the addition of $150 \mu \mathrm{l}$ DMSO (Sigma-Aldrich). Absorbance (A) was determined by measuring the absorbance at $490 \mathrm{~nm}$ using a Victor3 spectrofluorimeter (Perkin Elmer, Foster City, USA) at 24, 48 and 72 h post-transfection. Each assay was performed in triplicate and each experiment was repeated at least three times. Cell-growth curves were calculated as A mean values of triplicates per group.

Transwell assay. The migration assay was performed in a 6-well transwell chamber (Corning, Cambridge, MA, USA), which contained an $8 \mu \mathrm{m}$ pore size polycarbonate membrane filter for migration assay. Cells were trypsinized and suspended in a serum-free medium containing $1 \%$ bovine serum albumin at a concentration of $5 \times 10^{4}$ cells/insert. Medium supplemented with $10 \%$ fetal calf serum was added to the lower chamber. After reculturing with $5 \% \mathrm{CO}_{2}$ at $37^{\circ} \mathrm{C}$ for $24 \mathrm{~h}$, the transwell chambers were inverted and the cells were removed by swabbing and stained with crystal violet (Beyotime Institute of Biotechnology, Shanghai, China).

Statistical analysis. All statistical analyses were performed using SPSS 17.0 (SPSS, Chicago, USA). Data are presented as the mean \pm standard deviation. The differences of two groups and three groups were statistically analyzed using the Student's $t$-test and Analyze Compare Means One-Way ANOVA. The differences of ratios were statistically analyzed using the $\chi^{2}$ test. $\mathrm{P}<0.05$ was considered to indicate a statistically significant difference.

\section{Results}

Expression of USP9X is upregulated in hepatoma SMMC7721 and HepG2 cell lines. The protein expression of USP9X was detected in SMMC7721, HepG2 and L02 by western blot 
Table II. Protein level of USP9X in three cell groups $(n=3$, mean \pm standard deviation).

\begin{tabular}{lc} 
Groups & USP9X relative protein expression \\
\hline SMMC7721 & $0.53 \pm 0.03^{\mathrm{a}}$ \\
HepG2 & $0.47 \pm 0.05^{\mathrm{a}}$ \\
L02 & $0.18 \pm 0.03$ \\
\hline
\end{tabular}

${ }^{\mathrm{a}} \mathrm{P}<0.01$ compared with negative control group. USP9X, ubiquitin specific protease $9 \mathrm{X}$.

Table III. USP9X protein level in different treated SMMC7721 and HepG2 cells after siRNA interference $(n=3$, mean \pm standard deviation).

\begin{tabular}{lcc}
\hline Groups & SMMC7721 & HepG2 \\
\hline USP9X-siRNA & $0.21 \pm 0.01^{\mathrm{a}}$ & $0.36 \pm 0.01^{\mathrm{a}}$ \\
NC & $0.62 \pm 0.02$ & $0.94 \pm 0.01$ \\
\hline
\end{tabular}

${ }^{a} \mathrm{P}<0.01$ compared with $\mathrm{NC}$ group. NC, negative control; USP9X-siRNA, ubiquitin specific protease 9X-small interfering RNA.

Table IV. The apoptosis of different treated SMMC7721 and HepG2 cells $(n=3 ; \%$, mean \pm standard deviation).

\begin{tabular}{lcc}
\hline Groups & SMMC7721 & HepG2 \\
\hline USP9X-siRNA & $27.96 \pm 2.49^{\mathrm{a}}$ & $23.48 \pm 1.60^{\mathrm{a}}$ \\
NC & $15.02 \pm 3.03$ & $9.56 \pm 2.33$
\end{tabular}

${ }^{\mathrm{a}} \mathrm{P}<0.01$ compared with $\mathrm{NC}$ group. $\mathrm{NC}$, negative control; USP9X-siRNA, ubiquitin specific protease 9X-small interfering RNA.

analysis. The results suggested that the protein expression of USP9X was upregulated in SMMC7721 and HepG2 (Table II; $\mathrm{P}<0.01$ ).

USP9X expression was inhibited by siRNA in SMMC7721 and HepG2 cells. To examine the off-target effect of RNAi, USP9X-siRNA and NC were transfected into SMMC7721 and HepG2 cells using Lipofectamine 2000. USP9X expression was evaluated by western blot analysis. The results showed that USP9X-siRNA could effectively inhibit the expression of USP9X in SMMC7721 and HepG2 cell lines (Table III; $\mathrm{P}<0.01$ ).

USP9X-siRNA enhances SMMC7721 and HepG2 cell apoptosis. After SMMC7721 and HepG2 cells were transfected with USP9X-siRNA, cellular apoptosis was first examined by Annexin V and PI staining followed by FCM analysis. The percentage of apoptotic cells in the USP9X-siRNA group was higher than the NC group in both SMMC7721 and HepG2 (Figs. 1 and 2 and Table IV; $\mathrm{P}<0.01$ ).
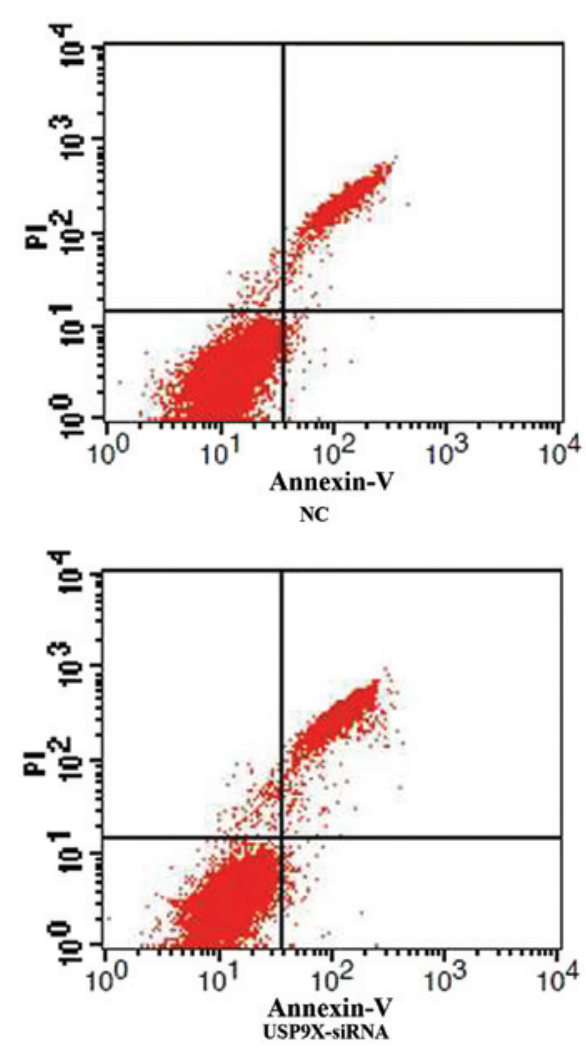

Figure 1. The apoptosis of differently treated groups in SMMC7721 by flow cytometry assay. The percentage of apoptotic cells in USP9X-siRNA group was higher than negative control group in SMMC7721 cells. USP9X-siRNA, ubiquitin specific protease 9X-small interfering RNA.
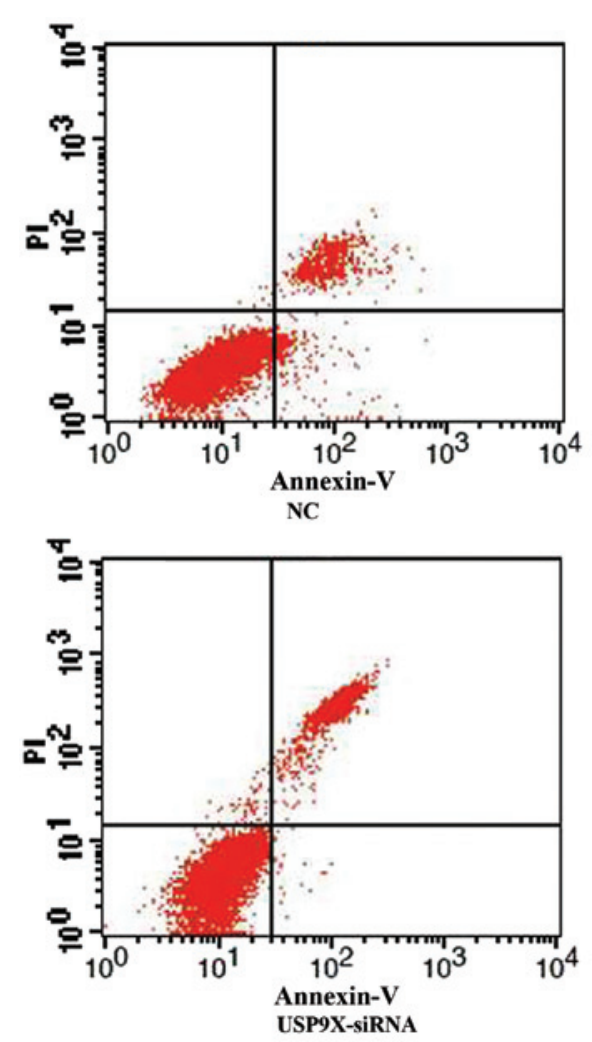

Figure 2. The apoptosis of different treated groups in HepG2 by flow cytometry assay. The percentage of apoptotic cells in USP9X-siRNA group was higher than negative control group in HepG2 cells. USP9X-siRNA, ubiquitin specific protease 9X-small interfering RNA. 
Table V. Comparison of cell absorbance in different treated SMMC7721 and HepG2 cells ( $\mathrm{n}=3$, mean \pm standard deviation).

\begin{tabular}{|c|c|c|c|c|c|c|}
\hline \multirow[b]{2}{*}{ Groups } & \multicolumn{3}{|c|}{ SMMC7721 } & \multicolumn{3}{|c|}{ HepG2 } \\
\hline & $24 \mathrm{~h}$ & $48 \mathrm{~h}$ & $72 \mathrm{~h}$ & $24 \mathrm{~h}$ & $48 \mathrm{~h}$ & $72 \mathrm{~h}$ \\
\hline USP9X-siRNA & $0.30 \pm 0.08^{\mathrm{a}}$ & $0.53 \pm 0.12^{\mathrm{a}}$ & $0.96 \pm 0.21^{\mathrm{a}}$ & $0.24 \pm 0.10^{\mathrm{a}}$ & $0.41 \pm 0.17^{\mathrm{a}}$ & $0.77 \pm 0.36^{\mathrm{a}}$ \\
\hline $\mathrm{NC}$ & $0.40 \pm 0.08$ & $0.72 \pm 0.12$ & $1.11 \pm 0.22$ & $0.32 \pm 0.10$ & $0.56 \pm 0.16$ & $0.88 \pm 0.34$ \\
\hline
\end{tabular}

${ }^{\text {a }}<<0.01$ compared with NC group. NC, negative control; USP9X-siRNA, ubiquitin specific protease 9X-small interfering RNA.

Table VI. Comparison of amounts of cell migration in different treated SMMC7721 and HepG2 cells $(\mathrm{n}=3$, mean \pm standard deviation).

\begin{tabular}{lcc}
\hline Groups & SMMC7721 & HepG2 \\
\hline USP9X-siRNA & $27.00 \pm 4.36^{\mathrm{a}}$ & $24.67 \pm 4.51^{\mathrm{a}}$ \\
NC & $55.70 \pm 4.04$ & $56.00 \pm 2.65$
\end{tabular}

${ }^{a} \mathrm{P}<0.01$ compared with NC group. NC, negative control; USP9X-siRNA, ubiquitin specific protease 9X-small interfering RNA.

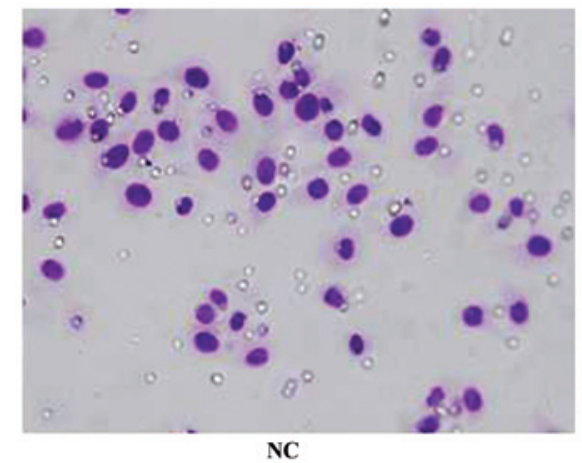

NC

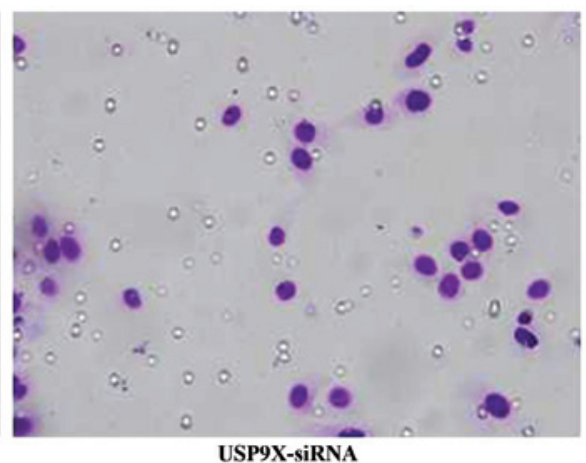

USP9X-siRNA

Figure 3. The cell migration amounts of differently treated groups in SMMC7721 by Transwell assay. The migration of SMMC7721 cells transfected with USP9X-siRNA was significantly reduced when compared with the cells of NC groups. NC, negative control. USP9X-siRNA, ubiquitin specific protease 9X-small interfering RNA.
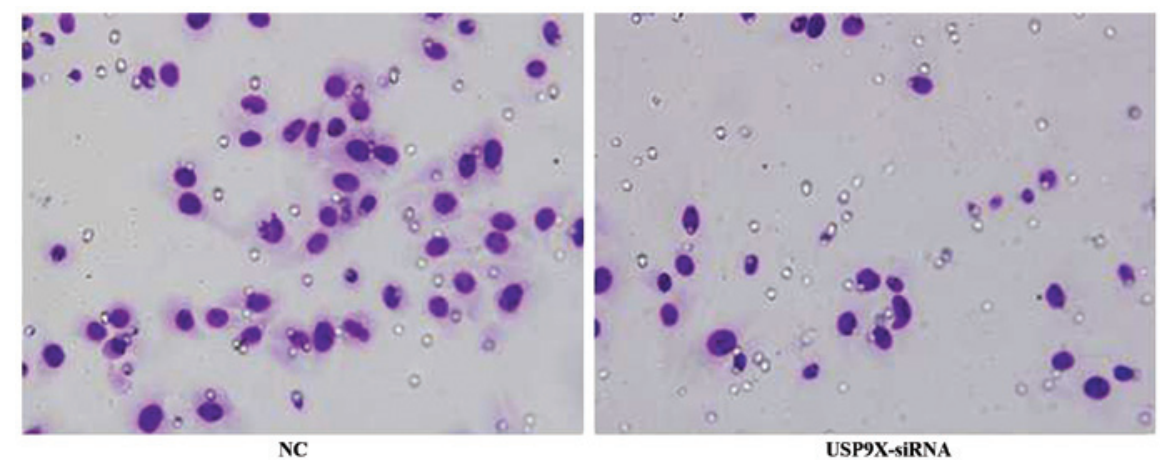

Figure 4. The cell migration amounts of differently treated groups in HepG2 by Transwell assay. The migration of HepG2 cells transfected with USP9X-siRNA was significantly reduced when compared with the cells of NC groups. NC, negative control. USP9X-siRNA, ubiquitin specific protease 9X-small interfering RNA.

USP9X-siRNA reduces SMMC7721 and HepG2 cell viability. As shown in Table V, the MTT assay revealed that the USP9X-siRNA group exhibited significantly reduced cell viability compared with the $\mathrm{NC}$ group cells $(\mathrm{P}<0.01)$.
USP9X-siRNA inhibits the migration of SMMC7721 and HepG2 cells. The results of the transwell assay revealed that the migration of SMMC7721 and HepG2 cells transfected with USP9X-siRNA was significantly reduced when compared with 
that of the $\mathrm{NC}$ groups $(\mathrm{P}<0.01)$, indicating that suppression of USP9X inhibited the migration of SMMC7721 and HepG2 cells (Figs. 3 and 4 and Table VI; $\mathrm{P}<0.01$ ).

\section{Discussion}

Recently, an increasing number of studies have demonstrated that USP9X is involved with cancer (5-14). USP9X expression was found to be higher in lung, colon and breast cancer, when compared with normal tissues in vitro $(5,10)$. Furthermore, in lung and colon cancers, decreased USP9X expression resulted in the promotion of cellular apoptosis in vivo (5), which indicated that USP9X expression may be a potential predictor of such cancers. However, at present, the association between USP9X expression and HCC has not been reported. Therefore, in the present study, the USP9X gene was knocked down in SMMC7721 and HepG2 cells using siRNA to study the potential effect of USP9X on HCC cells. In SMMC7721 and HepG2 cells, along with the effective silencing of USP9X by specific siRNA, cell viability and migration were reduced while cell apoptosis was increased.

Cell viability and apoptosis are important in the oncogenesis and chemotherapy resistance of HCC cells. This study demonstrated that the viability of SMMC7721 and HepG2 cells was reduced by USP9X-siRNA. The apoptosis ratio in the USP9X-siRNA group was significantly higher than in the NC group. This evidence indicates that USP9X is a crucial factor in HCC tumor growth. The current study also shows that cell migration was downregulated when USP9X expression was inhibited, indicating that USP9X-siRNA alone may inhibit cell migration. Additionally, USP9X-siRNA significantly reduced cell viability, which consequently resulted in a relative reduction in cell migration.

In conclusion, the results in the current investigation revealed that RNAi-mediated downregulation of USP9X effectively inhibits the growth and migration of SMMC7721 and HepG2 cells. However the mechanisms associated with USP9X regulating the bioactivity of HCC remain unknown. USP9X has been previously shown to bind to the Mcl-1 protein, which was reported to inhibit cell apoptosis in $\operatorname{HCC}(16,17)$, and inhibit proteasomal degradation in lymphoma (13). In other tumors, such as lung cancer, colon cancer and lymphoma, USP9X inhibited cancer cell apoptosis by influencing the degradation of specific protein Mcl-1 (5). Therefore we speculate that USP9X may inhibit cell apoptosis by influencing Mcl-1 in HCC cells. USP9X may be a potential target for the treatment and early detection of HCC, however, further studies are required to clarify the mechanism by which USP9X is involved in the development and progression of HCC, and its related pathway in HCC.

\section{Acknowledgements}

This study was supported by the National Natural Science Foundation of China (grant no. 81171562), the Natural Science Foundation of Chongqing, China (grant no. CSTC, 2013yykfA110010) and the Science Foundation of Yuzhong district, Chongqing, China (grant no. 20130118).

\section{References}

1. Llovet JM, Burroughs A and Bruix J: Hepatocellular carcinoma. Lancet 362: 1907-1917, 2003.

2. Marrero JA: Multidisciplinary management of hepatocellular carcinoma: where are we today? Semin Liver Dis 33 (Suppl 1): 3-10, 2013.

3. Yau T, Chan P, Epstein R and Poon RT: Evolution of systemic therapy of advanced hepatocellular carcinoma. World J Gastroenterol 14: 6437-6441, 2008.

4. El-Serag HB and Rudolph KL: Hepatocellular carcinoma: epidemiology and molecular carcinogenesis. Gastroenterology 132: 2557-2576, 2007.

5. Peddaboina C, Jupiter D, Fletcher S, et al: The downregulation of Mcl-1 via USP9X inhibition sensitizes solid tumors to Bcl-xl inhibition. BMC Cancer 12: 541, 2012.

6. DiAntonio A and Hicke L: Ubiquitin-dependent regulation of the synapse. Annu Rev Neurosci 27: 223-246, 2004.

7. Yang Bo and XIEcong Hua: Ubiquitin specific peptidase $9 \mathrm{X}$ and tumor. J Int Oncol 38: 900-902, 2011.

8. Kitagawa K, Kotake Y and Kitagawa M: Ubiquitin-mediated control of oncogene and tumor suppressor gene products. Cancer Sci 100: 1374-1381, 2009

9. Becuwe M, Herrador A, Haguenauer-Tsapis R, Vincent $\mathrm{O}$ and Léon S: Ubiquitin-mediated regulation of endocytosis by proteins of the arrestin family. Biochem Res Int 2012: 12, 2012.

10. Deng S, Zhou H, Xiong R, et al: Over-expression of genes and proteins of ubiquitin specific peptidases (USPs) and proteasome subunits (PSs) in breast cancer tissue observed by the methods of RFDD-PCR and proteomics. Breast Cancer Res Treat 104: 21-30, 2007

11. Sun H, Kapuria V, Peterson LF, et al: Bcr-Abl ubiquitination and Usp9X inhibition block kinase signaling and promote CML cell apoptosis. Blood 117: 3151-3162, 2011.

12. Kapuria V, Peterson LF, Fang D, Bornmann WG, Talpaz M and Donato NJ: Deubiquitinase inhibition by small-molecule WP1130 triggers aggresome formation and tumor cell apoptosis. Cance Res 70: 9265-9276, 2010.

13. Schwickart M, Huang X, Lill JR, et al: Deubiquitinase USP9X stabilizes MCL1 and promotes tumor cell survival. Nature 463: 103-107, 2010.

14. Xie Y, Avello M, Schirle M, et al: Deubiquitinase FAM/USP9X interacts with the E3 ubiquitin ligase SMURF1 protein and protects it from ligase activity-dependent self-degradation. J Biol Chem 288: 2976-2985, 2013.

15. Livak KJ and Schmittgen TD: Analysis of relative gene expression data using real-time quantitative PCR and the 2 (-Delta Delta C (T) ) method. Methods 25: 402-408, 2001.

16. Schulze-Bergkamen H, Fleischer B, Schuchmann M, et al: Suppression of Mcl-1 via RNA interference sensitizes human hepatocellular carcinoma cells towards apoptosis induction. BMC Cancer 6: 232, 2006.

17. Sieghart W, Losert D, Strommer S, et al: Mcl-1 overexpression in hepatocellular carcinoma: a potential target for antisense therapy. J Hepatol 44: 151-157, 2006. 